\title{
Mapa da Empatia em Saúde como instrumento de reflexão em cenário de ensino não assistencial
}

\author{
The Health Empathy Map as an instrument of reflection in a non-care teaching scenario \\ Luíza Uchôa de Resende Sousa' (D) |uchoaluiza@yahoo.com.br \\ Eliane Perlatto Moura² (1) elianeperlatto@gmail.com \\ José Maria Peixoto² ${ }^{2}$ jmpeixoto.prof@gmail.com \\ Janaina de Souza Aredes ${ }^{2}$ (1) janainaaredes@gmail.com \\ Camila do Carmo Said ${ }^{2}$ (D) camiladocarmosaid@gmail.com
}

\section{RESUMO}

Introdução: O desenvolvimento da empatia durante a graduação tem a finalidade de formar egressos com mais preparo na construção de uma boa relação com seu paciente. O Mapa da Empatia em Saúde (MES) é uma ferramenta adaptada com o propósito de desenvolver, por meio da autorreflexão, a empatia dos estudantes e futuros médicos.

Objetivo: Este estudo teve como objetivos avaliar o efeito do uso do MES na empatia autorrelatada em estudantes de Medicina no cenário de grupo tutorial (GT) e analisar as características das reflexões realizadas.

Método: Trata-se de estudo quase experimental, com abordagem de métodos mistos, na modalidade plano explicativo, realizado com 59 estudantes do terceiro ano da Unifenas de Belo Horizonte. Quarenta e um estudantes utilizaram o MES no GT, e 18 fizeram o GT de forma habitual. No início do estudo, os participantes responderam a um questionário sociodemográfico e à Escala de Empatia de Jefferson (JSPE-Br), a qual foi reaplicada ao final. Realizaram-se análises estatísticas em que se compararam os escores da JSPE-Br entre os grupos e as fases do estudo, e fez-se ainda a análise de conteúdo do MES.

Resultado: O escore global de empatia se mostrou elevado em todas as fases e não diferiu entre os grupos. A análise de conteúdo revelou que o MES estimulou os estudantes a refletir sobre o quadro dos pacientes, com base nos pilares da empatia, a partir da leitura de suas narrativas. As reflexões variaram de aspectos biomédicos a perspectivas socioafetivas complexas.

Conclusão: O MES estimulou a reflexão empática dos estudantes em cenário não assistencial e foi capaz de identificar a abrangência das reflexões, o que possibilitou as diferentes perspectivas a serem discutidas aqui.

Palavras-chave: Educação Médica; Empatia; Estudantes de Medicina.

\section{ABSTRACT}

Introduction: The development of empathy during graduation aims at forming graduates who are more prepared to build a good relationship with their patients. The health empathy map (HEM) is an adapted tool with the purpose of developing empathy in students and future doctors using self-reflection.

Objectives: To evaluate the effect of using the HEM, in the tutorial group scenario, on the empathy score measured by the Jefferson scale and in the assessment of the students' empathic relationship with their patients.

Method: This was a quasi-experimental study that used a mixed-method approach, in the explanatory plan modality carried out with 56 students from the $5^{\text {th }}$ semester of the undergraduate medical course at José do Rosário Vellano University, UNIFENAS-BH. The students were divided into two groups: G1: students who used the HEM in the tutorial group (TG) strategy and G2: students who did not use HEM in the TG. All students completed a sociodemographic questionnaire at the beginning of the study and the Jefferson Empathy Scale at the beginning and the end of the study. Statistical analyses were performed comparing the JSPE-Br scores between the groups and the study phases, and the HEM content analysis was performed.

Results: The global empathy score was high in all phases of the study and did not differ between the groups. The content analysis revealed that the HEM encouraged students to reflect on the patients' conditions, based on the mainstays of empathy, and considering the reading of their narratives. The reflections ranged from the biomedical aspects to complex socio-affective perspectives.

Conclusion: HEM stimulated the students' empathic reflection in a non-care setting and was able to identify the scope of the reflections, allowing the different perspectives to be discussed.

Keywords: Medical Education; Empathy; Medical Students.

${ }^{1}$ Centro Universitário Presidente Tancredo de Almeida Neves, São João Del Rei, Minas Gerais, Brasil.

2 Universidade José do Rosário Vellano, Belo Horizonte, Minas Gerais, Brasil.

Editora-chefe: Rosiane Viana Zuza Diniz.

Editor associado: Roberto Zonato Esteves.

Recebido em 25/05/21; Aceito em 19/08/21.

Avaliado pelo processo de double blind review. 


\section{INTRODUÇÃO}

A empatia é uma habilidade social que envolve a capacidade de colocar-se no lugar do outro, de modo a fornecerIhe apoio e levá-lo a sentir-se compreendido'. É um construto multidimensional com componentes afetivos, cognitivos e comportamentais ${ }^{2,3}$. Por meio do componente afetivo, compartilham-se os estados emocionais dos outros, enquanto os componentes cognitivos e comportamentais são responsáveis pela capacidade de raciocinar sobre os estados mentais de outras pessoas e deliberar as ações de comunicação e ajuda4.

Na área de saúde, a empatia se destaca nas discussões sobre o aprimoramento das relações interpessoais objetivando a humanização das práticas assistenciais ${ }^{5,6}$, além de ser associada a melhores resultados terapêuticos e maior satisfação dos pacientes e profissionais ${ }^{7}$. Apesar de sua importância, tem sido constatado que a empatia pode sofrer mudanças ao longo do curso de Medicina, o que significa uma variação de pequeno aumento a diminuição ${ }^{8}$.

O desenvolvimento da habilidade de empatia é um dos objetivos essenciais de aprendizagem na formação de profissionais da saúde, e seu aprimoramento deve ocorrer desde a graduação9. Vários recursos têm sido utilizados para potencializar o desenvolvimento da empatia em estudantes de Medicina ${ }^{10-13}$. No entanto, grande parte das estratégias empregadas restringe-se ao componente cognitivo, mas não ao construto multidimensional.

Estudos indicam que o desenvolvimento da habilidade de empatia nos estudantes é mais eficaz quando o tema é inserido no contexto das atividades acadêmicas. Uma das estratégias idealizadas para o ensino da empatia em cenários da saúde é o Mapa da Empatia em Saúde (MES), que favorece a apropriação conceitual da empatia. O MES foi elaborado com a finalidade de estimular a autorreflexão dos estudantes e dar suporte à prática da empatia ${ }^{14}$. Estudo conduzido por Cançado et al. ${ }^{15}$ demonstrou aumento da percepção da empatia médica pelos pacientes após a utilização do MES, sugerindo que esse instrumento favoreceu o desenvolvimento do comportamento empático por parte dos médicos residentes.

Existem evidências de que a leitura pode ser utilizada para o desenvolvimento da empatia ${ }^{16}$, pois exige a imaginação das diversas perspectivas de seus personagens em acontecimentos não testemunhados ${ }^{17}$. Este estudo teve por objetivo avaliar o efeito do uso do MES durante a leitura de casos clínicos (sem a presença do paciente) no grupo tutorial (GT), estratégia adotada em modelos curriculares da aprendizagem baseada em problemas.

\section{MÉTODOS}

Trata-se de estudo quase experimental, com abordagem de métodos mistos, na modalidade plano explicativo, no qual análises qualitativas contribuem para explicar resultados quantitativos iniciais ${ }^{18}$.

A amostra foi constituída por estudantes de Medicina do quinto período, da Universidade José do Rosário Vellano (Unifenas) de Belo Horizonte, em Minas Gerais, que cursavam o Bloco de Síndromes Cardiológicas, durante o primeiro semestre de 2019, no cenário do GT. Incluíram-se estudantes regularmente matriculados que concordaram em participar do estudo e assinaram o Termo de Consentimento Livre e Esclarecido (TCLE). A opção pelo quinto período baseou-se em estudos que identificaram ser o terceiro ano do curso, que marca a transição para o ciclo clínico, um momento em que se observa o início da redução da empatia em estudantes de Medicina ${ }^{11}$.

O componente quantitativo do estudo foi realizado em duas fases:

- Fase 1: inicialmente, todos os estudantes foram orientados a preencher um questionário com questões sociodemográficas e a Escala de Empatia de Jefferson, versão brasileira para estudantes (Jeferson Scale of Physician Empathy - JSPE-Br) ${ }^{19}$.

- Fase 2: nas aulas subsequentes, formaram-se dois grupos. No grupo intervenção (G1), os estudantes foram orientados a preencher individualmente - MES após a sessão de análise do caso clínico no GT. No grupo controle (G2), os participantes frequentaram as sessões de GT normalmente e não precisaram preencher o MES.

Os questionários e os mapas foram identificados pelo nome e número de matrícula do estudante, e os mapas também continham o número do caso clínico. Ao final das cinco semanas do bloco, os participantes preencheram novamente a JSPE- $\mathrm{Br}$ durante a aula de encerramento (Figura 1).

No desenvolvimento da pesquisa, utilizaram-se três instrumentos:

- Questionário sociodemográfico: trata-se de um questionário semiestruturado, com 26 questões, para identificação e caracterização de fatores comportamentais, de saúde, familiares e do ambiente de ensino.

- JSPE-Br: trata-se de um instrumento de autorrelato que avalia o nível de empatia em estudantes de Medicina. É composta por 20 perguntas mensuradas pela escala de Likert: de um ("discordo totalmente") a sete ("concordo totalmente"). Não existe um ponto de corte na avaliação produzida; a pontuação é gradativa, e, quanto maior o escore obtido, mais empático seria o estudante avaliado ${ }^{19}$. 
- MES: esse instrumento possui quatro quadrantes que contemplam os três componentes da empatia: tomada de perspectiva, compartilhamento emocional e preocupação empática. Cada quadrante contém uma pergunta: 1. "O que você sentiria se estivesse no lugar desta pessoa?"; 2. "Qual a sua percepção das necessidades e desejos desta pessoa, atuais e futuros?"; 3. "Como me sinto conhecendo a história desta pessoa?"; 4. "Como posso ajudar esta pessoa?". No centro do MES, consta o desenho de um emoji sem as sobrancelhas e a boca, e abaixo há seis emojis representando as expressões faciais básicas ${ }^{14}$. Os estudantes foram orientados a preencher o MES, responder às perguntas $e$, ao final, indicar a expressão facial que, segundo eles, representava o sentimento do paciente descrito no caso do GT.

Neste estudo, utilizaram-se os sete casos clínicos já existentes no Bloco Síndromes Cardiológicas. Os problemas se relacionam aos grandes temas da cardiologia, sendo dois de hipertensão arterial, um de angina estável, um de síndrome coronariana aguda, dois de insuficiência cardíaca e um de fibrilação atrial. São apresentados por meio de vinhetas clínicas que abordam aspectos biomédicos e apresentam considerações psicossociais relacionadas à pessoa e aos familiares que figuram no caso.

Este estudo foi aprovado pelo Comitê de Ética em Pesquisa da Unifenas: Certificado de Apresentação para Apreciação Ética (CAAE) n 02657618.8.0000.5143.

\section{Análise dos dados}

Para a análise dos dados quantitativos, realizou-se estatística descritiva conduzida por análise univariada, em que se utilizaram o teste t de Student e/ou a Análise de Variância (ANOVA), o teste qui-quadrado, o teste exato de Fisher e a análise de regressão linear múltipla. O nível de significância utilizado foi de $5 \%(p<0,05)$. Adotou-se o programa SPSS 14.0 for Windows (software estatístico).

Para integrar e complementar os resultados obtidos, foi realizada a análise qualitativa do MES, por meio da técnica de análise de conteúdo, modalidade categorial, proposta por Bardin ${ }^{20}$ que se baseia na decodificação de um texto em diversos elementos, os quais são classificados e formam agrupamentos temáticos que auxiliam na compreensão e descrição do conteúdo das mensagens.

Primeiramente, os mapas foram transcritos na íntegra em arquivos do Microsoft Excel. As respostas contidas em cada mapa foram digitadas em tabelas (separadas por cada caso clínico) conforme a estrutura geral do MES (quatro quadrantes).

Organizaram-se os dados circunscritos nos mapas a partir da identificação de ideias-chave oriundas das respostas ${ }^{20}$, restritas aos registros contidos em cada quadrante dos mapas e de acordo com as características da reflexão empática demonstradas pelo estudante na escrita. No quadrante 1, foi considerado se o estudante refletiu sobre o caso, colocandose no lugar do paciente (sim ou não). No quadrante 3 , levouse em conta se o estudante foi afetado pelo caso ao conhecer a história do paciente (sim ou não). Nos quadrantes 2 e 4, as respostas dos estudantes foram classificadas nos seguintes

Figura 1. Etapas do estudo.

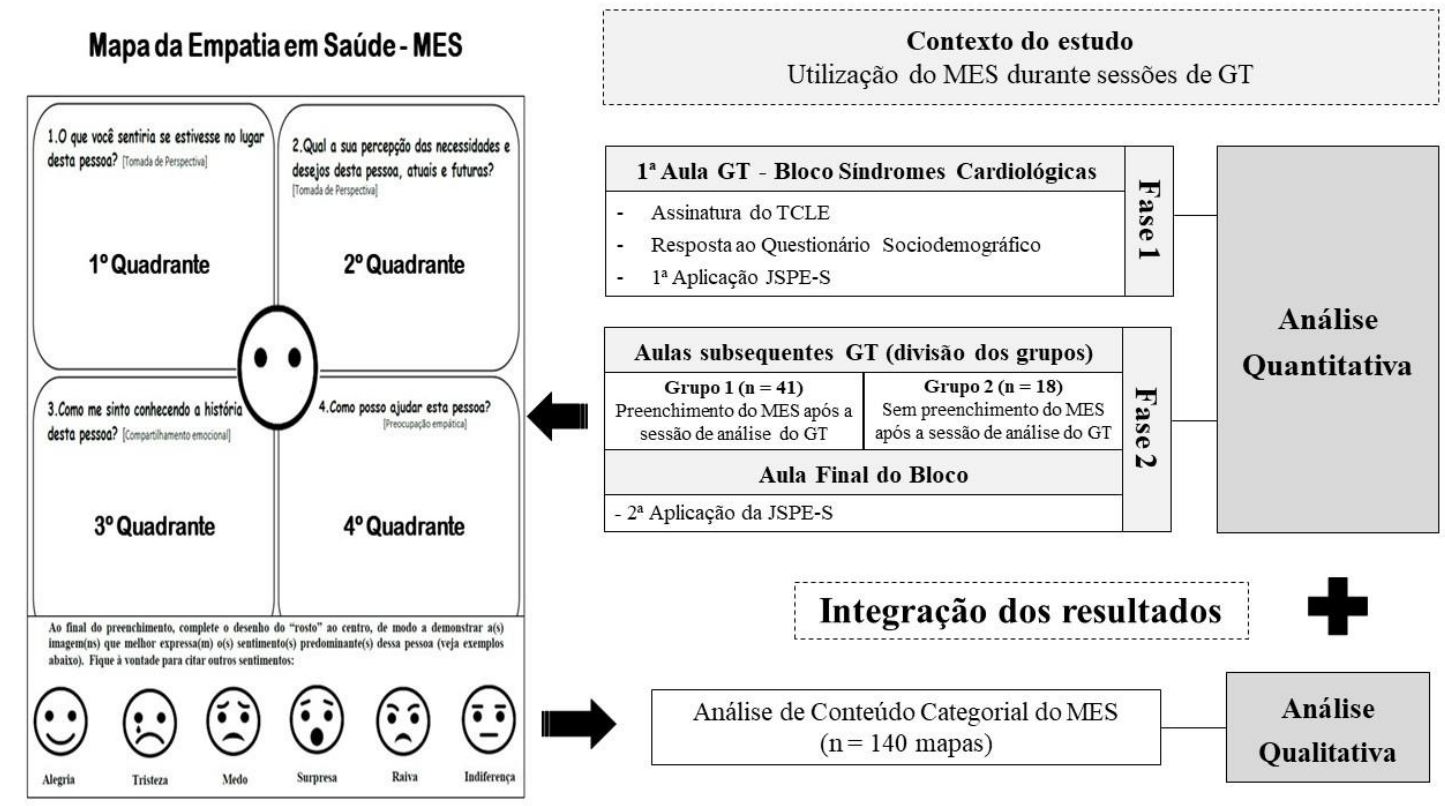

GT: grupo tutorial. 
eixos: identificação de necessidades restritas à doença e/ou aspectos biomédicos; considera os sentimentos e desejos da pessoa; e considera o contexto familiar e/ou social da pessoa.

Para garantir a validação e confiabilidade dos dados, essa classificação foi realizada de forma independente por três pesquisadores do estudo. Definiram-se as codificações finais por meio de reuniões da equipe de pesquisa, durante as quais discrepâncias foram discutidas até que o consenso fosse alcançado.

Com a finalidade de interpretação dos dados obtidos a partir da análise de conteúdo, desenvolveu-se um modelo de classificação das respostas para cada quadrante do MES (Quadro 1), utilizando-se como referenciais teóricos os estágios de desenvolvimento da tomada de perspectiva na infância proposto por Selman ${ }^{21}$ e a classificação dos níveis de empatia de Fuchs ${ }^{22}$. Selman ${ }^{21}$ propõe que na infância a habilidade de tomada de perspectiva evolui em estágios, nos quais a criança, inicialmente, não diferencia suas perspectivas das dos outros (de 4 a 5 anos), posteriormente percebe que as perspectivas das pessoas podem diferir (de 6 a 7 anos), passa a reconhecer que outras perspectivas podem ser corretas (de 8 a 10 anos), para então compreender que as perspectivas podem se relacionar a grupos de pessoas (de 10 a 12 anos), e finalmente que não se limitam às relações interpessoais, mas também ao sistema social. Apesar de a proposta de Selman ${ }^{21}$ se relacionar à infância, observa-se, em sua classificação, que as perspectivas podem diferir entre as pessoas e se relacionar a um grupo social.

Fuchs $^{22}$ descreve que a empatia apresenta dois níveis distintos em relação à forma de manifestação: 1. a empatia primária, que seria uma forma implícita, na qual as emoções observadas nos outros são sentidas e experimentadas pelo observador, o que ele denomina de ressonância corporal; e 2. a empatia estendida, uma forma explícita em que o observador imagina como seria estar no lugar do outro, refletindo e fazendo inferências, o que o autor denomina de transposição imaginária, uma forma que proporciona a possibilidade de uma compreensão social de nível superior ${ }^{23}$.

Assim, o modelo de classificação proposto neste estudo se baseou nas características evolutivas das perspectivas de Selman ${ }^{21}$, na proposta de classificação de Fuchs ${ }^{22}$, que pode ser aplicada a todas as idades, e na habilidade do estudante em identificar a perspectiva do paciente em níveis progressivos de suas relações (níveis 1, 2 e 3): 1. quando o aluno demonstra observar apenas os aspectos biomédicos relacionados à sua prática profissional; 2. quando considera os componentes psicoemocionais do indivíduo; e 3. quando é capaz de considerar as relações sociais envolvidas na perspectiva do paciente (Quadro 1).

\section{RESULTADOS}

\section{Caracterização da amostra}

Participaram do estudo 59 estudantes por se enquadrarem nos critérios de inclusão propostos. Em relação ao perfil sociodemográfico dos participantes: $55,9 \%(n=33)$ do sexo feminino; 55,2\% ( $n=32$ ) com idade entre 19 e 21 anos; 69,5\% ( $n$ $=41)$ autodeclarados de raça branca; 79,7\% $(n=47)$ adeptos de alguma religião; $96,6 \%(n=57)$ solteiros; $89,8 \%(n=53)$ cursaram o ensino médio em escola particular; $67,8 \%(n=40)$ não possuíam bolsa estudantil; 42,4\% ( $n=25)$ tinham renda familiar acima de R\$ $15.000,00 ; 96,6 \%(n=57)$ não possuíam atividade remunerada; $86,4 \%(n=51)$ possuíam algum tipo de passatempo; $74,6 \%(n=44)$ não praticavam atividade artística; $66,1 \%(n=39)$ frequentavam

Quadro 1. Modelo de classificação das respostas por quadrantes do MES em dimensões da empatia.

\begin{tabular}{|c|c|c|}
\hline Quadrantes do MES & Características das respostas & Dimensões da empatia \\
\hline $\begin{array}{l}\text { Quadrante } 3 \\
\text { Como me sinto conhecendo a história desta } \\
\text { pessoa? } \\
\text { [compartilhamento emocional] }\end{array}$ & $\begin{array}{l}\text { Sentimentos e reflexões externados nas respostas } \\
\text { indicam se o estudante foi afetado ao conhecer o } \\
\text { caso do paciente. Se a história do outro "repercutiu" } \\
\text { no estudante. }\end{array}$ & $\begin{array}{l}\text { Empatia primária } \\
\text { Ressonância corporal }\end{array}$ \\
\hline $\begin{array}{l}\text { Quadrante } 1 \\
\text { O que você sentiria se estivesse no lugar } \\
\text { desta pessoa? } \\
\text { [tomada de perspectiva] }\end{array}$ & $\begin{array}{l}\text { Demonstram que o estudante se colocou ou não no } \\
\text { lugar do outro. Sentimentos/sensações e reflexões } \\
\text { externados nas respostas indicam que o estudante } \\
\text { se colocou no lugar do paciente. }\end{array}$ & $\begin{array}{l}\text { Empatia estendida } \\
\text { Transposição imaginária }\end{array}$ \\
\hline \multirow{2}{*}{$\begin{array}{l}\text { Quadrante } 2 \\
\text { Qual a sua percepção das necessidades e } \\
\text { desejos desta pessoa, atuais e futuras? } \\
\text { [tomada de perspectiva] }\end{array}$} & $\begin{array}{l}\text { Nível 1: Demonstram identificação de necessidades } \\
\text { com foco restrito à doença e/ou aspectos } \\
\text { biomédicos. }\end{array}$ & \multirow{3}{*}{$\begin{array}{l}\text { Empatia estendida } \\
\text { Compreensão superior }\end{array}$} \\
\hline & $\begin{array}{l}\text { Nível 2: Considera os desejos, os sentimentos e as } \\
\text { necessidades expressos pelo paciente descritos na } \\
\text { vinheta clínica. }\end{array}$ & \\
\hline $\begin{array}{l}\text { Como posso ajudar esta pessoa? } \\
\text { [preocupação empática] }\end{array}$ & $\begin{array}{l}\text { Nível 3: Considera o contexto familiar (demais } \\
\text { atores envolvidos no caso) e/ou o contexto social } \\
\text { (emprego, arrimo de família etc.). }\end{array}$ & \\
\hline
\end{tabular}

Fonte: Elaborado pelos autores com base na análise de conteúdo do MES e nos referenciais teóricos propostos por Selman ${ }^{21}$ e Fuchs ${ }^{22}$. 
eventos artísticos; 79,7\% ( $n=47)$ não apresentavam doença crônica; $74,6 \%(n=44)$ tinham história de doença crônica familiar; e $88,1 \%(n=52)$ declararam a Medicina como o primeiro curso superior. Os dois grupos eram homogêneos em relação às variáveis sociodemográficas analisadas.

\section{Escore de empatia nos grupos analisados}

A média do escore global geral de empatia dos participantes foi elevada em todas as fases do estudo (Tabela 1). Não se observaram diferenças nos escores obtidos entre os grupos, em cada domínio da escala e nem dentro de um mesmo grupo, nas distintas fases do estudo ( $p>0,05$ - ANOVA). Entretanto, os estudantes de ambos os grupos apresentaram valores significativamente inferiores no domínio "capacidade de colocar-se no lugar do outro" em comparação aos outros domínios da escala nas duas fases do estudo ( $p=0,040$ - teste de Friedman) (Tabela 2).

Observou-se que os estudantes com história familiar de doença crônica apresentaram médias dos escores de empatia maiores do que aqueles que não possuíam, tanto no escore geral ( $p=0,033$ - Mann-Whitney) quanto no domínio compaixão ( $p=0,029$ - Mann-Whitney). As demais variáveis sociodemográficas não apresentaram correlações aos escores obtidos pela JSPE-Br.

\section{Análise de conteúdo dos Mapas de Empatia em Saúde}

Considerou-se o total de 140 mapas que contemplaram sete casos clínicos sendo: caso $1(n=23)$; caso $2(n=23)$; casos
3 e 4 (complementares $-n=31)$; caso $5(n=25)$; caso $6(n=20)$; e caso $7(\mathrm{n}=18)$. A análise foi realizada para cada caso e cada quadrante do MES.

Para avaliar o componente afetivo, consideraram-se as respostas que demonstraram sentimentos e reflexões do estudante ao conhecer o caso e a história vivenciada pelo paciente, obtidas no terceiro quadrante do MES - "Como me sinto conhecendo a história desta pessoa?". Trata-se da empatia primária vinculada à ressonância corporal, conforme classificação proposta neste estudo (Quadro 1).

Para o componente cognitivo, foram analisadas as respostas que demonstraram como o estudante imaginava o que sentiria se estivesse no lugar do paciente, obtidas no primeiro quadrante do MES - "O que você sentiria se estivesse no lugar desta pessoa?". Trata-se da empatia expandida na qual ocorre a transposição imaginária (Quadro 1).

A maioria dos estudantes, $72,9 \%(n=102)$, conseguiu identificar os próprios sentimentos diante da situação do paciente, entretanto $27,1 \%(n=38)$ demonstraram dificuldade em expressar o compartilhamento emocional (Gráfico 1):

"Sinto que pacientes como o senhor Eduardo são pessoas que não se preocupam tanto com a própria saúde, mas que fazem de tudo para os outros" (E2 - caso 2).

Em relação à empatia estendida, $95,7 \%(n=134)$ dos estudantes demonstraram ter refletido o que sentiriam se estivessem no lugar do paciente (transposição imaginária)

Tabela 1. Média de escores globais de empatia dos estudantes por grupo e fase do estudo obtidos pela Escala de Jefferson.

\begin{tabular}{ccccccc}
\hline \multirow{2}{*}{ Participantes } & \multirow{2}{*}{} & Fase & \multicolumn{5}{c}{ Medidas descritivas } \\
\cline { 4 - 7 } & & 1 & Mínimo & Máximo & Média & dp \\
\hline \multirow{2}{*}{ Grupo 1 } & \multirow{2}{*}{41} & 2 & 5,3 & 6,6 & 6,1 & 0,4 \\
& & 1 & 4,6 & 6,7 & 6,0 & 0,5 \\
\hline \multirow{2}{*}{ Grupo 2 } & \multirow{2}{*}{18} & 2 & 5,3 & 6,6 & 5,9 & 0,4 \\
& & & 4,9 & 6,7 & 5,8 & 0,5 \\
\hline
\end{tabular}

Tabela 2. Médias dos escores de empatia dos estudantes, entre os domínios da Escala de Jefferson, por grupo e fase do estudo.

\begin{tabular}{|c|c|c|c|c|}
\hline \multirow{4}{*}{ DOMÍNIOS } & \multicolumn{4}{|c|}{ GRUPO / FASE } \\
\hline & \multicolumn{2}{|c|}{ GRUPO 1} & \multicolumn{2}{|c|}{ GRUPO 2} \\
\hline & FASE 1 & FASE 2 & FASE 1 & FASE 2 \\
\hline & Média $\pm d p$ & Média \pm dp & Média $\pm d p$ & Média $\pm d p$ \\
\hline Tomada de perspectiva & $5,9 \pm 0,5$ & $5,9 \pm 0,5$ & $5,8 \pm 0,3$ & $5,8 \pm 0,6$ \\
\hline Compaixão & $6,2 \pm 0,3$ & $6,1 \pm 0,5$ & $5,9 \pm 0,5$ & $5,9 \pm 0,6$ \\
\hline Capacidade de colocar-se no lugar do outro & $4,2 \pm 1,6$ & $4,1 \pm 1,5$ & $4,4 \pm 1,5$ & $4,4 \pm 1,4$ \\
\hline $\mathrm{p}$ & $<0,001$ & 0,006 & $<0,001$ & 0,040 \\
\hline
\end{tabular}

O valor da probabilidade de significância $(p)$ refere-se ao teste de Friedman. 
Gráfico 1.Distribuição das respostas segundo os componentes afetivo e cognitivo da empatia, obtidas nos 140 mapas avaliados.

\section{Foi afetado pelo caso? (compartilhamento emocional)}

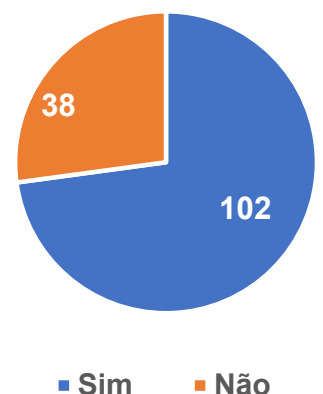

(Gráfico 1), e medo, angústia, tristeza e preocupação foram os sentimentos mais citados nas respostas:

"Me sentiria com medo e angustiada com tantos sinais e sintomas que foram piorando ao longo do caminho que percorria" (E7 - caso 5).

Em relação à compreensão superior da empatia estendida, foram analisados os quadrantes 2 ("Qual a sua percepção das necessidades e desejos desta pessoa, atuais e futuras?") e 4 ("Como posso ajudar esta pessoa?) do MES. No quadrante 2 , observou-se que $50 \%$ das respostas demonstraram uma percepção com foco restrito à doença:"Sinto que o paciente necessita de auxílio para tratar os seus sintomas" (E9 - caso 5). Algumas respostas $(22,1 \%)$ consideraram os sentimentos e desejos do paciente, e em 15\% delas somente os sentimentos do paciente foram expressos:

"Vejo que essa paciente tem esperança de dias melhores e uma boa visão de futuro almeja crescer e se aprimorar" (E15 - caso 6).

Já 7,1\% das respostas consideraram as dimensões da doença e os sentimentos do paciente:

"Ele deseja ajuda para tratar seus sintomas, pois se sente muito preocupado e com medo de ser algo mais grave e levá-lo a óbito" (E14 - caso 7).

O contexto social e/ou familiar foi considerado em $25,7 \%$ das respostas, das quais $3,6 \%$ consideraram somente a dimensão social:

"A paciente não estava preocupada consigo mesma e, sim, com o que o seu 'adoecer' ou até mesmo como sua morte traria de impacto para sua família que depende dela" (E10 - caso 1).

Dos participantes, $15 \%$ consideraram as dimensões doença e social:

"Melhorar a saúde para cuidar dos filhos e mãe" (E9caso 1).

\section{Refletiu sobre o caso? (transposição imaginária)}

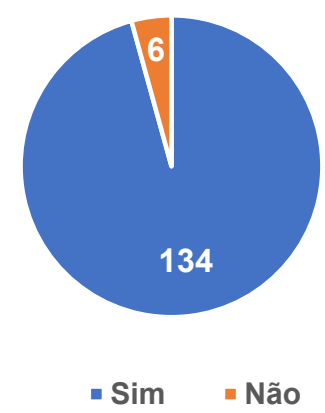

Os níveis pessoa e social foram considerados por 4,3\%:

"Acredito que nesse momento é com as pessoas que ele vai deixar, se preocupando com seus entes queridos. Dessa forma, é importante trazê-los para o cuidado de forma a acalmar o paciente e ajudá-lo a resolver pendências" (E10 - caso 5).

E somente 2,9\% consideraram os níveis 1 (doença e/ou aspectos biomédicos), 2 (componentes psicoemocionais) e 3 (contexto social e familiar) simultaneamente:

"O paciente sente-se com medo devido à possibilidade de perder a visão. Sente-se preocupado por ter que trabalhar e pagar a faculdade do filho" (E3 - caso 2).

Ainda, ao refletirem sobre como poderiam ajudar o paciente (quadrante 4 ), $42,8 \%$ das respostas mantiveram o foco somente na doença:

"Posso explicar para ele como funciona o tratamento medicamentoso e mostrar a importância do mesmo. Assim, possivelmente o paciente irá aderir ao tratamento e ter uma condição de saúde controlada" (E13 - caso 2).

Dos participantes, $42,8 \%$ consideraram os sentimentos e desejos do paciente ao refletirem sobre o modo como poderiam ajudá-lo, e 12,8\% levaram em conta o nível pessoa:

"Me colocando em seu lugar, escutando seus medos e tentando dar o amparo necessário nesse momento" (E8 - caso 5)

E 30\% consideraram os níveis doença e pessoa:

"Auxiliando no tratamento e no apoio emocional para fazê-lo aderir e se sentir seguro ao me ter como seu médico" (E7 - casos 3 e 4).

Somente $19 \%$ dos estudantes refletiram sobre o contexto social e/ou familiar do paciente no momento de decidir como poderiam auxiliá-lo, e 1,4\% refletiu somente sobre o nível social: "Aconselhar sobre resolver assuntos inacabados $e$ conversar com a família" (E1 - caso 5). 
Dos estudantes, $2,9 \%$ consideraram os níveis doença e social:

"Apoio familiar. E, antes disso, uma forma de tratar e ajudar" (E11 - caso 5).

\section{E 2,9\% consideraram os níveis pessoa e social:}

"Se estivesse no momento em que ele partir, falaria que ajudaria a família dele no que eles precisassem e que ele fora um bom homem e chegara ao fim da vida com dignidade" (E9-caso 5).

Apenas $6,4 \%$ dos estudantes consideraram os três níveis - doença, pessoa e social - ao refletirem sobre o que poderiam fazer para ajudar o paciente:

"A encaminharia para um psicólogo para que haja um apoio emocional em relação à situação do divórcio, do etilismo, do cuidado com a mãe dependente. Reforçaria MEV [mudança de estilo de vida] para ela melhorar a qualidade de vida dela. Medicações" (E10 - caso 6).

\section{DISCUSSÃO}

Os resultados deste estudo demonstraram níveis elevados dos escores de empatia geral obtidos pela JSPE$\mathrm{Br}$ nos participantes, nas duas fases da pesquisa. Resultados semelhantes foram encontrados por Caires $^{24}$ em estudo na mesma instituição.

É interessante observar que, neste estudo, apesar de os escores se apresentarem elevados, a subescala que avalia a "capacidade de colocar-se no lugar do outro" foi reduzida para toda a amostra independentemente da fase da pesquisa. Resultados semelhantes foram obtidos por Mohammadi et al ${ }^{25}$ e Díaz-Narváez ${ }^{26}$ que avaliaram estudantes de medicina e odontologia.

Esses dados são intrigantes, uma vez que a habilidade da empatia pressupõe a habilidade de se colocar no lugar do outro. Segundo Decety et al. ${ }^{27}$, esse domínio está inserido na esfera da tomada de perspectiva. Do ponto de vista psicométrico, foi considerado um fator residual na escala original, reforçando o baixo poder discriminatório dos níveis de empatia da escala de Jefferson ${ }^{28-30}$.

A empatia tem uma dimensão cognitiva e outra emocional, sendo a cognitiva mais fácil de ser aferida por escalas ${ }^{31}$. No entanto, é o componente afetivo que mobiliza a disposição para o cuidado compassivo que, por sua vez, é influenciado por questões socioculturais e idiossincráticas. Por essas razões, a avaliação da empatia requer a associação de instrumentos metodológicos com abordagens distintas, como já apontado por Ren et al. ${ }^{32}$ e Berg et al. ${ }^{33}$. Por tratar-se de um construto cuja análise é complexa e multidimensional, a incorporação de instrumentos qualitativos tem o potencial de oferecer contribuições adicionais ao entendimento da empatia e à sua própria avaliação em distintos cenários de ensino e aprendizagem.

O MES utilizado no contexto do GT, no qual o aluno tem contato com a descrição de casos clínicos, foi capaz de estimular reflexões sobre os próprios sentimentos do estudante, caso estivesse no lugar do paciente, despertando o componente emocional da empatia: a capacidade de ser afetado por emoções do outro. Essa capacidade de avaliar os seus próprios sentimentos é particularmente importante, uma vez que, segundo Dymond ${ }^{34}$, a transposição imaginativa de si no pensamento, no sentimento ou na ação do outro é componente fundamental da tomada de perspectiva, que influencia a natureza das experiências emocionais empáticas.

Moll et al. ${ }^{35}$ consideram a tomada de perspectiva como uma das mais importantes habilidades sociocognitivas, uma vez que reflete uma compreensão geral de que um mesmo evento pode ser visto e interpretado de várias maneiras, dependendo do ponto de vista daquele que observa. Para Rogers ${ }^{36,37}$, uma das forças mais poderosas em uma relação é a capacidade de captar com precisão os sentimentos e significados pessoais que o paciente está vivendo.

Identificou-se, ainda, que alguns estudantes têm dificuldade de refletir sobre os seus próprios sentimentos em relação à situação do paciente. Essa reflexão se faz importante em um mundo no qual as emoções vêm sendo consideradas estorvos e elementos distratores em uma prática médica tecnicista ${ }^{16}$. D'Andrea ${ }^{38}$, ao apresentar algumas dificuldades para o estabelecimento de relação empática, aponta a incapacidade do médico de reconhecer-se como pessoa total, com consciência dos próprios sentimentos, e o fato de ser a medicina centralizada mais em si mesma que no indivíduo.

A reflexão sobre os sentimentos próprios e os do paciente exemplifica o domínio da compaixão que compõe o construto da empatia. Weingartner et al. ${ }^{39}$ definem compaixão como o reconhecimento do sofrimento emocional de si próprio ou dos outros, associado ao desejo de reduzir tal sofrimento, e a associa a resultados positivos na saúde do paciente, além de melhorar sua satisfação.

A análise do conteúdo dos mapas revelou ainda que uma porcentagem expressiva dos estudantes considerou somente a doença do paciente, e poucos levaram em conta os três níveis da empatia: doença, sentimento e contexto social. Esses dados apontam para a necessidade de desenvolvimento de estratégias educacionais que objetivem ampliar a compreensão do estudante sobre o paciente.

Observa-se que, apesar de os escores de empatia geral dos estudantes pela JSPE-Br apresentarem-se elevados, a análise de suas repostas pelo MES demonstrou que eles tiveram dificuldades em alguns dos componentes da 
empatia, como o estabelecimento das necessidades dos pacientes e da empatia compassiva, em que prevaleceu uma atenção predominantemente centrada na doença, já que os participantes do estudo não foram capazes de identificar as perspectivas relacionadas aos sentimentos advindos do processo de adoecer.

Este estudo apresenta algumas limitações, como o fato de ter sido realizado no início do terceiro ano, antes de as atividades assistências terem sido iniciadas. Isso pode ter impedido o encontro de valores mais reduzidos para os escores da escala JSPE-Br; afinal, estudos indicam que a queda desses escores tem ocorrido a partir terceiro ano. Outra limitação está relacionada ao fato de tratar-se de uma avaliação transversal realizada em um único momento do curso e apenas concernente aos conteúdos relacionados à cardiologia, o que não permite transferir seus achados para os estudantes do início do curso e os concluintes, nem para outras áreas temáticas da medicina.

No entanto, o estudo apresenta importantes contribuições para a compreensão do desenvolvimento da empatia nas profissões da saúde, em que a utilização de métodos mistos permitiu um elemento de validação da consistência entre os dados obtidos. Trata-se do primeiro estudo desenvolvido com o MES em cenário de ensino não assistencial que utilizou da leitura de casos clínicos como estratégia para estimular a imaginação e a reflexão empática dos estudantes de Medicina. $O$ estudo demonstrou que a análise do conteúdo das reflexões dos estudantes no MES capturou nuances que não seriam identificadas por meio das escalas de autorrelatos comumente utilizadas nos estudos de empatia.

O MES foi imprescindível porque explicitou aos educadores os pontos em que os estudantes precisam receber suporte para que possam evoluir na capacidade de tomada de perspectiva em níveis mais complexos. O presente estudo apresenta ainda uma proposta para a categorização da tomada de perspectiva para as áreas de saúde, que pode ser utilizada como referência para a análise dos conteúdos do MES; afinal, são poucas as tentativas de categorização em adultos.

\section{CONSIDERAÇÕES FINAIS}

Com base nos resultados apresentados, podemos inferir que o MES é um instrumento com potencial para estimular o desenvolvimento da empatia também em ambientes não assistenciais, utilizando-se da leitura de casos clínicos como fonte estimuladora para a imaginação e reflexão sobre os vários pontos de vista da relação médico-paciente. A análise de conteúdo do MES foi capaz de capturar nuances sobre habilidade de tomada de perspectiva dos estudantes não identificadas pela escala de autorrelato JSPE-Br. Os resultados do estudo mostraram ainda que o MES pode ser uma ferramenta útil para avaliar a empatia em estudantes, sendo capaz de apontar as diferenças de dimensões de empatia entre os estudantes e ressaltar pontos específicos que podem ser trabalhados de forma mais individualizada ou em grupo.

\section{CONTRIBUIÇÃO DOS AUTORES}

Luíza Uchôa de Resende Sousa participou do delineamento da pesquisa, da coleta de dados, da discussão dos resultados e da redação do artigo. Eliane Perlatto Moura participou do delineamento da pesquisa, da coleta de dados, da discussão dos resultados, da redação e revisão do artigo, além de orientar todo o processo. José Maria Peixoto, Janaina de Souza Aredes e Camila do Carmo Said participaram do delineamento da pesquisa, da coleta de dados, da discussão dos resultados e da redação e revisão do artigo.

\section{CONFLITO DE INTERESSES}

Declaramos não haver conflito de interesses.

\section{FINANCIAMENTO}

Declaramos não haver financiamento.

\section{REFERÊNCIAS}

1. Thomazi L, Moreira FG, De Marco MA. Avaliação da evolução da empatia em alunos do quarto ano da graduação em medicina da Unifesp em 2012. Rev Bras Educ Med. 2014;38(1):87-93.

2. Decety J, Jackson P. La arquitectura funcional de la empatía humana. Behav Cogn Neurosci Rev. 2004;3(2):71-100

3. Falcone EMDO, Pinho VD, Ferreira MC, Fernandes CS, D'Augustin JF, Krieger $S$, et al. Validade convergente do Inventário de Empatia (IE). Psico USF. 2013;8(2):203-9.

4. Thompson NM, Uusberg A, Gross JJ, Chakrabarti B. Empathy and emotion regulation: an integrative account. Prog Brain Res. 2019;247:273-304.

5. Costa FDD, Azevedo RCSD. Empatia, relação médico-paciente e formação em medicina: um olhar qualitativo. Rev Bras Educ Med. 2010;34(2):261-9.

6. Batista NA, Lessa SS. Aprendizagem da empatia na relação médicopaciente: um olhar qualitativo entre estudantes do internato de escolas médicas do Nordeste do Brasil. Rev Bras Educ Med. 2019;43(1):349-56.

7. De Benedetto MAC, Moreto G, Janaudis MA, Levites MR, Blasco PG. Educando as emoções para uma atuação ética: construindo o profissionalismo médico. Rev Bras Med. 2014; 71(n. esp. $m$ 2):15-24.

8. Ponnamperuma G, Yeo SP, Samarasekera DD. Is empathy change in medical school geo-socioculturally influenced? Med Educ. 2019;53(7):655-65.

9. Nascimento HCF, Ferreira Júnior WA, Silva AMTC, Carvalho IGM, Bastos GCFC, Almeida RJ. Análise dos níveis de empatia de estudantes de medicina. Rev Bras Educ Med. 2018;42(1):152-60.

10. Suartz CV, Quintana MI, Lucchese AC, De Marco MA. Avaliação de empatia em residentes de especialidades clínicas e cirúrgicas da Universidade Federal de São Paulo. Rev Bras Educ Med. 2013;37(3):320-5.

11. Moreto G, Blasco PG. A Erosão da empatia nos estudantes de Medicina: um desafio educacional. Rev Bras Med. 2012;69(1):12-7.

12. Torres AR, Smairal SI, Vellozol AP, Trench EV, Lovadinil GB, Lima MCP. Ensinando a anamnese psiquiátrica para estudantes de medicina através da inversão de papéis: relato de experiência. Rev Bras Educ Med. 2019;43(2):200-7. 
13. Vogel $D$, Meyer $M$, Harendza S. Verbal and non-verbal communication skills including empathy during history taking of undergraduate medical students. BMC Med Educ. 2018;18(1):157-64.

14. Peixoto JM, Moura EP. Mapa da Empatia em Saúde: elaboração de um instrumento para o desenvolvimento da empatia. Rev Bras Educ Med. 2020;44(1):e029.

15. Cançado PVR, Moura EP, Peixoto JM. O efeito do Mapa da Empatia em Saúde no comportamento empático médico percebido pelo paciente. Saude e Pesqui. 2021;14(2):e9081.

16. Medeiros NS, Santos TR, Trindade EMV, Almeida KJQD. Avaliação do desenvolvimento de competências afetivas e empáticas do futuro médico. Rev Bras Educ Med. 2013;37(4):515-25.

17. Prestes $Z$, Tunes E. A trajetória de obras de Vygotsky: um longo percurso até os originais. Estud Psicol. 2012;29(3):327-40.

18. Creswell JW, Clark VL. Pesquisa de métodos mistos. 2a ed. Porto Alegre: Penso; 2013.

19. Paro HB, Daud-Gallotti RM, Tiberio IC, Pinto RM, Martins MA. Brazilian version of the Jefferson Scale of Empathy: psychometric properties and factor analysis. BMC Med Educ. 2012;1:12-73.

20. Bardin L. Análise de conteúdo. São Paulo: Edições 70; 2015.

21. Selman RL. The relation of role taking to the development of moral judgment in children. Child Dev. 1971;42:79-91.

22. Fuchs T. Levels of empathy-primary, extended, and reiterated empathy. In: Lux V., Weigel S, editors. Palgrave studies in the theory and history of psychology. Empathy: epistemic problems and cultural-historical perspectives of a cross-disciplinary concept. London: Palgrave Macmillan; 2017. p. 27-47.

23. Schmidsberge $F$, Löffler-Stastka $H$. Empathy is proprioceptive: the bodily fundament of empathy - a philosophical contribution to medical education. BMC Med Educ. 2018;18(1):1-6.

24. Caires VV. Análise da empatia no estudante de medicina da Faculdade de Medicina - Unifenas-BH ao longo da graduação [dissertação]. Belo Horizonte: Universidade José do Rosário Vellano; 2019.

25. Mohammadi A, Kamali K, Jahandizi MH. Level of empathy for patients among students of Zanjan University of Medical Sciences in 2016. Preventive Care in Nursing and Midwifery Journal. 2016-2017;6(4):38-46.

26. Díaz-Narváez V, Oyarzún-Muñoz M, Reyes-Reyes A, Calzadilla-Núñez A, Martínez PT, González-Valenzuela C, et al. Psychometry and empathy levels and its dimensions in postgraduate students of dental specialties. Eur J Dent Educ. 2021.
27. Decety J, Cowell JM. The complex relation between morality and empathy. Behav Cogn Neurosci Rev. 2014;18(7):337-9.

28. Velicer WF, Fava JL. Effects of variable and subject sampling on factor pattern recovery. Psychol. Methods. 1998;3(2):231-51.

29. Hojat M, Gonnella JS. What matters more about the interpersonal reactivity index and the Jefferson Scale of Empathy? Their underlying constructs or their relationships with pertinent measures of clinical competence and patient outcomes? Acad Med. 2017;92(6):743-75.

30. Nunes RAA. Perfil da empatia médica de residentes do $3^{\circ}$ ano de ortopedia e traumatologia [dissertação]. Belo Horizonte: Universidade José do Rosário Vellano; 2018.

31. Ickes W, Marangoni C, García S. Studying empathic accuracy in a clinically relevant context. In: Ickes W, editor. Empathic accuracy. New York: Guilford; 1997. p. 282-310.

32. Ren GSG, Min JTY, Samarasekera DD. Complex and novel determinants of empathy change in medical students. Korean J Med Educ. 2016;28(1):67-78.

33. Berg K, Blatt B, Lopreiato J, Jung J, Schaeffer A, Heil D, et al. Standardized patient assessment of medical student empathy. Acad Med. 2015;90(1):105-11.

34. Dymond RF. Personality and empathy. J Consult Psychol. 1950;14(5):343-50.

35. Moll $H$, Meltzoff AN. Perspective-taking and its foundation in joint attention. In: Roessler J, Lerman $\mathrm{H}$, Eilan $\mathrm{N}$, editors. Perception, causation, and objectivity. Oxford, UK: Oxford Scholarship Online; 2011. p. 286-304.

36. Rogers CR. Definições das noções teóricas. In: Rogers CR, Kinget MS Psicoterapia e relações humanas: teoria e prática da terapia não-diretiva. Belo Horizonte: Interlivros; 1977. p. 54-82.

37. Rogers CR. Um jeito de ser. São Paulo: EPU, 1983 [access in 30 aug 2019]. Available from: https://www.ufpe.br/documents/38958/229603/ ata/5b609c0d-0f53-4450-a2f8- 012c41b95428?version=1.0.

38. D'Andrea FF. Desenvolvimento da personalidade. São Paulo. Difusão Européia do livro, Edusp; 1972.

39. Weingartner LA, Sawning S, Shaw MA, Klein JB. Compassion cultivation training promotes medical student wellness and enhanced clinical care. BMC Med Educ. 2019;19(1):139. 